\title{
ESTUDIO EXPERIMENTAL DE LA EXPRESIÓN DE LAS PROTEÍNAS DE LA PARED VESICAL EN LA OBSTRUCCIÓN DEL TRACTO URINARIO INFERIOR EN EL CONEJO
}

\author{
J. SALINAS CASADO(1), M. VIRSEDA CHAMORRO ${ }^{(2)}$, A. MARTÍN VEGA ${ }^{(3)}$, \\ A. HERNÁNDEZ LAO(4), J.A. HERRERO PAYO(5)
}

${ }^{(1)}$ Servicio de Urología. Hospital Clinico San Carlos. Universidad Complutense. Madrid. (2)Servicio de Urología. Hospital Central de la Defensa. Madrid. (3)Departamento de Bioquímica. Hospital Clínico San Carlos. Universidad Complutense. Madrid. ${ }^{(4)}$ Servicio de Urología. Clínica Puerta de Hierro. Madrid. ${ }^{(5)}$ Servicio de Urología. Hospital Severo Ochoa. Leganés (Madrid).

Actas Urol Esp. 28 (5): 341-349, 2004

\section{RESUMEN}

ESTUDIO EXPERIMENTAL DE LA EXPRESIÓN DE LAS PROTEÍNAS DE LA PARED VESICAL EN LA OBSTRUCCIÓN DEL TRACTO URINARIO INFERIOR EN EL CONEJO

OBJETIVOS: Determinar la relación de los niveles tisulares de las proteínas contráctiles: actina total, beta-actina, alfa-actina sarcomérica y caldesmón y de la "Heat Stress Protein" HSP-70, con la obstrucción parcial del tracto urinario inferior.

MATERIAL Y MÉTODOS: Se realizó un estudio experimental en 43 conejos machos neozelandeses que fueron divididos en un grupo control y seis grupos experimentales a los que se les realizó obstrucción parcial del tracto urinario inferior mediante ligadura de la uretra. Los grupos experimentales se definieron por el día del sacrificio de los animales que tuvo lugar a las 24 horas, 7 días, 15 días, 1 mes, 3 meses y 6 meses de la obstrucción. En todos los animales se determinó la concentración en la pared de la capa muscular de actina total, beta-actina, alfa-actina sarcomérica, caldesmón y HSP-70, mediante antisueros y electroforesis según la técnica del "Western Blotting”. Los resultados fueron analizados mediante ANOVA, utilizando el test de contraste a posteriori de Scheffe.

RESULTADOS: El peso de las vejigas de los animales obstruidos aumentó significativamente en dos etapas: hasta el día 15, y entre el día 15 y el final del estudio, correspondiendo a las fases de hipertrofia inicial y compensación, respectivamente. El nivel total de actina mostró una disminución significativa al inicio de la fase de compensación, mientras que la beta-actina aumentó en la fase de hipertrofia inicial, y la alfa-actina sarcomérica al final de la fase de compensación, mientras que el caldesmón y la HSP-70 lo hicieron al final de la fase de hipertrofia inicial.

CONCLUSIONES: Del análisis de datos se deduce que la determinación de los niveles de caldesmón es el marcador que mejor se ajusta a los cambios asociados a la obstrucción parcial del tracto urinario inferior en el conejo.

PALABRAS CLAVE: Obstrucción parcial del tracto urinario inferior. Hipertrofia vesical. Actina. Caldesmón. "Heat Shock Proteins".

\section{ABSTRACT \\ EXPERIMENTAL STUDY OF THE EXPRESSION OF THE BLADDER WALL PROTEINS IN BLADDER OUTLET OBSTRUCTION IN THE RABBIT}

OBJECTIVES: To determine the relationship of the bladder wall levels of the contractile proteins: whole actin, beta-actin, alpha-sarcomeric-actina caldesmon and of the Heat Stress Protein HSP-70, with the partial bladder outlet obstruction.

MATERIAL AND METHODS: It was carried out an experimental study on 43 New-Zealand rabbits. These animals were divided into a control group and six experimental groups with partial bladder outlet obstruction induced by an incomplete tie around the urethra. The experimental groups were defined according the day when the animals were sacrified: at 24 hours, 7 days, 15 days, 1 month, 3 months and 6 months of the obstruction. It was determined in all animals the muscular bladder wall concentration of whole actin, beta-actin, alpha-sarcomeric-actin, caldesmon and HSP-70 protein, according to Western Blotting technique. The results were analyzed by means of ANOVA, using the Scheffe post hoc method.

RESULTS: The bladder weight of the obstructed animals increased significantly in two stages: until the day 15 and between the day 15 and the end of the study, corresponding to theoretical phases of initial hypertrophy and compensation. The level of whole actin showed a significant decrease at the beginning of compensation phase, while the beta-actin level increased in the phase of initial hypertrophy and the alpha-sarcomeric-actin level increased at the end of the compensation phase, while the caldesmon and the HSP-70 made it at the end of the initial hypertrophy phase.

CONCLUSIONS: Analysing our data we conclued that the determination of the caldesmon levels is the marker that better adjusts to the changes associated with partial bladder outlet obstruction in the rabbit.

KEY WORDS: Partial bladder outlet obstruction. Bladder hypertrophy. Actin. Caldesmon. Heat Shock Proteins.

*Este trabajo ha sido patrocinado por una Beca de Investigación de la Asociación Española de Urología. 
$\mathrm{L}^{2}$ a hiperplasia benigna prostática (HBP) es un proceso muy frecuente en los varones de más de 50 años. En un porcentaje elevado de pacientes la HBP produce sintomas urinarios funcionales que varían desde el aumento de la frecuencia miccional nocturna, hasta la retención urinaria ${ }^{1}$.

Se admite que los sintomas producidos por la HBP se deben frecuentemente a la obstrucción del tracto urinario inferior ${ }^{2}$. La obstrucción del tracto urinario inferior produce cambios funcionales en la vejiga urinaria. La intensidad de la contractilidad vesical aumenta proporcionalmente al grado de obstrucción, aunque existe una pérdida de la capacidad vesical para mantener la contracción ${ }^{3}$. Esta disminución de la capacidad vesical para mantener la contracción sería responsable de la aparición de un volumen residual post-miccional ${ }^{4}$. Además la obstrucción urinaria crónica puede llegar a alterar la fase de llenado, favoreciendo la disminución de la acomodación vesical o capacidad de la vejiga de almacenar orina a baja presión ${ }^{5}$.

Para conocer los cambios histológicos, biomecánicos, y bioquímicos que tienen lugar en la vejiga urinaria como consecuencia de la obstrucción del tracto urinario inferior, se han realizado estudios experimentales en animales de laboratorio en los que se reproduce la obstrucción urinaria asociada a la HBP, mediante obstrucción parcial de la uretra con ligadura, manguito o anillo ${ }^{6}$.

Estos estudios han comprobado que la vejiga sufre alteraciones tanto de sus propiedades biomecánicas pasivas, como de sus propiedades contráctiles, que se correlacionan con una serie de cambios bioquímicos a nivel nuclear, citoplasmático y metabólico. Los cambios citoplasmáticos incluyen la alteración de la concentración de las proteínas del tejido conectivo y muscular (proteínas contráctiles), y la detección de proteínas habitualmente no presentes en la célula nor$\mathrm{mal}^{7,8}$.

Entre las proteínas específicas de la capa muscular vesical se encuentran la actina, que presenta varias isoformas y es responsable junto con la miosina de la contracción muscular, y el caldesmón que interviene en la adherencia de los fragmentos de actina y miosina, origen de la contracción tónica característica de las fibras musculares lisas ${ }^{9}$. También se ha descrito la apari- ción de proteínas de stress como la "Heat Shock Protein" de aproximadamente 70 KiloDaltons de peso molecular (HSP-70). Estas proteínas aparecerían en situaciones de lesión tisular como la obstrucción urinaria ${ }^{10}$. La determinación de la expresión de este tipo de proteínas podría ser un marcador bioquímico importante para determinar la presencia de obstrucción urinaria. El estudio de la variación de su expresión en relación al tiempo en que se mantiene la obstrucción urinaria inferior, permitiría determinar si existe correlación entre los niveles de dichas proteínas y el tiempo de obstrucción.

De confirmarse esta relación en este estudio básico experimental, una futura etapa sería identificar estas proteínas en varones con hiperplasia benigna prostática, mediante biopsia de la pared vesical, en orden a determinar la importancia clínica de estos marcadores.

El objetivo de nuestro estudio es determinar la variación con el tiempo de los niveles de las proteínas del tejido muscular: actina (en tres isoformas: alfa-actina sarcomérica, actina citoplasmática y beta-actina), caldesmón y de la proteína reactiva HSP-70, en las vejigas de animales de laboratorio sometidos a obstrucción parcial de la uretra.

\section{MATERIAL Y MÉTODOS}

Se realizó un estudio experimental utilizando 43 conejos neozelandeses machos adultos de peso medio 2.892 gramos (desviación típica: 874 gramos). Los conejos fueron divididos en dos grupos:

- Grupo control, formado por diez conejos. Estos conejos fueron anestesiados con ketamina (25 mg/10 mg i.m.) y mantenida con pentobarbital (i.v.) y sometidos a laparotomía abdominal y cierre de la misma. De estos conejos, uno fue sacrificado el mismo día de la intervención y sirvió como patrón de medida del resto de los conejos. Seis conejos se sacrificaron a la semana de la intervención y los otros tres a las dos semanas.

- Grupo experimental, formado por 33 conejos, anestesiados por el mismo procedimiento y sometidos a laparotomía media y obstrucción parcial de la uretra, según técnica descrita por Zhao et al. ${ }^{10}$ : cateterización uretral con sonda 8 Ch, ligadura alrededor del catéter con seda de 
$3 / 0$ y retirada de la sonda. Al día siguiente, a los 7 días, 15 días, 1 mes, 3 meses y 6 meses de la intervención los conejos fueron sacrificados. El número de conejos sacrificados en cada periodo fue de 4 (1 día), 6 (7 días), 6 (15 días), 7 (1 mes), 6 ( 3 meses) y 4 (6 meses).

\section{Tratamiento de las muestras}

Una vez sacrificados los conejos, las vejigas extraídas de los diferentes grupos, fueron etiquetadas y congeladas a $-80^{\circ} \mathrm{C}$. A partir de esos órganos se aisló la capa muscular y se cortaron pequeñas piezas de aproximadamente 3 gramos. Estas piezas fueron homogeneizadas en una solución tampón de extracción compuesta por 10 $\mathrm{mM}$ de Trisacetato, $10 \mathrm{mM}$ de NaCL, $1 \mathrm{mM}$ de EDTA (ácido etilendiamín tetra-acético), $1 \mathrm{mM}$ de PMSF (fenilmetilsulfonilfluorido) y $2 \mu \mathrm{M}$ de leupeptin. Para homogeneizar la muestra se añadieron $3 \mathrm{ml}$ de solución por gramo de tejido y se utilizó un homogeneizador Polytron-Kinetica PT $10^{\circledR}$ (Brinkmann, Westbury, NY), durante dos minutos bajo condiciones de temperatura cercanas a los $4^{\circ} \mathrm{C}$. El producto de la homogeneización fue centrifugado a 3900 x g durante 10 minutos. El sobrenadante obtenido fue distribuido en alícuotas de $30 \mu \mathrm{l}$, etiquetado y almacenado a $-80^{\circ} \mathrm{C}$.

\section{Cuantificación de proteínas}

Se cuantificó la masa total de proteínas de las alícuotas a través de un ensayo comercial basado en el método de Bradford ${ }^{11}$. Una vez cuantificadas las proteínas se eligió la masa total de proteínas en función de la muestra que tenía menor concentración de proteínas, que correspondió a $2,18 \mu \mathrm{g} / \mu \mathrm{l}$, con el fin de que todas las muestras tuvieran la misma cantidad. Se calculó el volumen extraído de cada muestra con el fin de que su concentración proteica fuera de $2,18 \mu \mathrm{g} / \mu \mathrm{l}$, y a continuación se añadió una solución de solución tampón salino fosfato (PBS) hasta llegar a $\operatorname{los} 10 \mu \mathrm{l}$ de volumen. Posteriormente se añadieron $20 \mu 1$ de solución tampón hasta llegar a un volumen total de $30 \mu l$.

\section{Electroforesis}

Las muestras fueron hervidas a $100^{\circ} \mathrm{C}$ durante 1 minuto, centrifugadas durante 2 minutos, posteriormente mantenidas en hielo. La solución tampón de desplazamiento ("running buffer") utilizada estaba compuesta por glicina 0,2 M y SDS al $0,1 \%$ en Tris $0,025 \mathrm{M}$ a pH 8,3. Las condiciones de electroforesis fueron de $10 \mathrm{~mA}$ de intensidad durante 2 horas, a temperatura ambiente. En todas las electroforesis se reservó una muestra como marcador de peso molecular, para confirmar que la proteína separada era la buscada en relación con su peso molecular.

\section{Transferencia de proteinas}

Después de la separación electroforética de las proteínas, éstas se transfirieron a una membrana de nitrocelulosa de acuerdo al procedimiento descrito por Burnette ${ }^{12}$, conocido como "Western Blotting”. Antes de completar la electroforesis, la solución tampón de transferencia compuesta por $25 \mathrm{mM}$ de Tris, $192 \mathrm{mM}$ de glicina y metanol al $20 \%, \mathrm{pH} 8,3$, fue preparada y mantenida a $4^{\circ} \mathrm{C}$. Las membranas de nitrocelulosa, que eran del mismo tamaño que el gel, los papeles de filtro y las esponjas se mantuvieron durante $15-20$ minutos en la solución de transferencia a $4^{\circ} \mathrm{C}$, antes de comenzar el proceso. La transferencia se realizó en cámara fría $\left(4^{\circ} \mathrm{C}\right)$, a $30 \mathrm{~V}$ durante más de 15 horas.

\section{Inmunoanálisis}

Después de la transferencia, la membrana de nitrocelulosa fue bañada en solución tampón de bloqueo, compuesta por PBS en leche en polvo desnatada al 5\% y Tween 20 al $0,1 \%$, durante una hora y suavemente agitada. Después del bloqueo se lavó la membrana tres veces durante 10 minutos con una solución de lavado compuesta por PBS y Tween 20 al 0,1\% (TPBS).

La membrana de celulosa fue incubada con solución tamponada salina, conteniendo $0,1 \%$ de TPBS, durante más de 15 horas con el anticuerpo específico en cámara fría $\left(4^{\circ} \mathrm{C}\right)$. Se lavó tres veces en solución de lavado y agitación suave durante 10 minutos, y fue incubada por segunda vez con el anticuerpo anti-IgG conjugado con peroxidasa (Anti Mouse IgG peroxidase conjugate $n^{\circ}$ A-4416 Sigma Chemical Company ${ }^{\circledR}$ ), diluido en PBS a la concentración de trabajo durante 3 horas con agitación suave a temperatura ambiente. Posteriormente, fue lavada con TPBS, 3 veces, durante 10 minutos con agitación suave. 
Los anticuerpos utilizados fueron:

- Anticuerpo monoclonal anti-actina ( $n^{\circ}$ producto: A-4700 Sigma Chemical Company ${ }^{\circledR}$ ). Dilución de trabajo: 1:500.

- Anticuerpo monoclonal anti-actina sarcomérica ( $\mathrm{n}^{\circ}$ producto: A-2172, Sigma Chemical Company $^{\circledR}$ ). Dilución de trabajo: 1:500.

- Anticuerpo monoclonal anti- $\beta$-actina (producto $\mathrm{n}^{\mathrm{o}}$ A-5441, Sigma Chemical Company ${ }^{\circledR}$ ). Dilución de trabajo: 1:5000.

- Anticuerpo monoclonal anti-caldesmón (producto $n^{\circ}$ C-6542, Sigma Chemical Company ${ }^{\circledR}$ ). Dilución de trabajo: 1:100.

- Anticuerpo monoclonal anti-HSP-70 (producto $\mathrm{n}^{\mathrm{o}} \mathrm{H}-5147$, Sigma Chemical Company ${ }^{\circledR}$ ). Dilución de trabajo: 1:5000.

Después de la segunda incubación, la membrana fue sumergida en $30 \mathrm{ml}$ de solución de sustrato de peroxidasa y sometida a agitación suave. La reacción enzimática producida es de tipo colorimétrico, siendo detenida posteriormente mediante la inmersión de la membrana en agua destilada (Fig. 1).

\section{Cuantificación densitométrica}

Las bandas obtenidas en la membrana mediante la reacción colorimétrica descrita anteriormente, fueron cuantificadas a través del programa de densitometría óptica "Quantity One" del densitómetro GS-710 de Bio-Rad ${ }^{\circledR}$. De las posibilidades analíticas del programa se tomaron en consideración la media de la densidad óptica, para la cuantificación de las concentraciones de anticuerpos anti-actina alfa sarcomérica, actina total, beta-actina y HSP-70, y el volumen ajustado para la cuantificación del anticuerpo anti-caldesmón, debido a que presentaban valores más homogéneos.

Se tomó como unidad estándar, el valor de la densidad óptica y el volumen ajustado de la vejiga del conejo control de 24 horas (valor 1), al que se refirieron el resto de las muestras analizadas. De esta manera, se evita el sesgo producido por las variaciones de concentración debidas a los diferentes tiempos de realización del registro.

\section{Análisis estadístico}

Los valores de las distintas muestras fueron almacenados en una base de datos Excell $^{\circledR}$ y

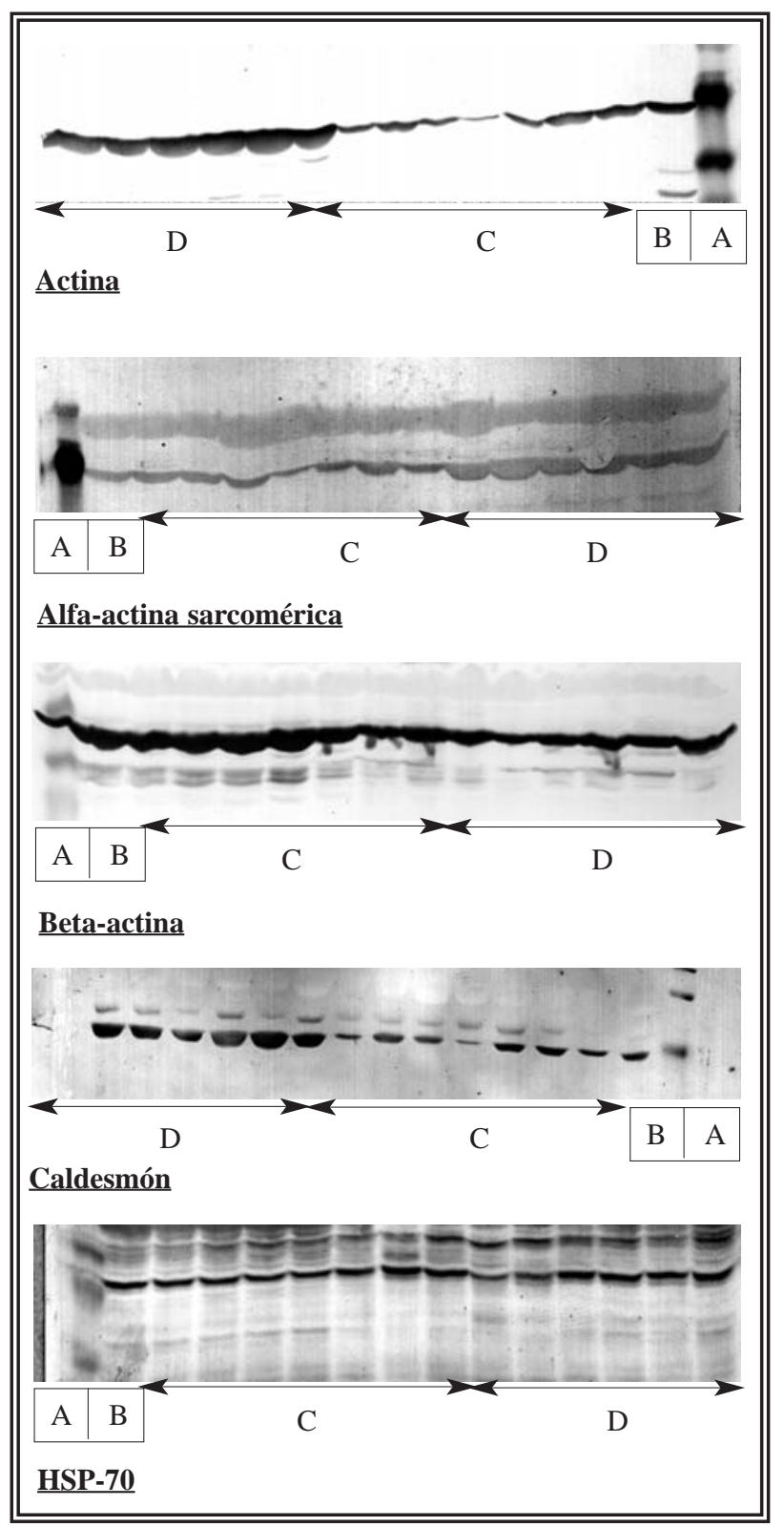

FIGURA 1. A: Bandas correspondientes al marcador de pesos moleculares. B: Banda correspondiente a la muestra control. C: Bandas correspondientes a los animales obstruidos durante 1 mes. D: Bandas correspondientes a los animales obstruidos durante 3 meses.

posteriormente importados al programa informático SPSS para su procesamiento. Se utilizó el procedimiento de análisis de la varianza (ANOVA) para determinar la significación de variación de la concentración de proteínas con el tiempo y el test de contraste a posteriori de Scheffe para comparar el nivel de proteínas en las diferentes etapas. El nivel de significación se fijó en $\mathrm{p}<0,05$ bilateral. 


\section{RESULTADOS}

La variación del peso de la vejiga con el tiempo de obstrucción se muestra en la Figura 2. El análisis de la varianza demostró una variación significativa del peso vesical con el tiempo $(\mathrm{p}=0,000)$. El test de contraste a posteriori de Sheffe demostró diferencias significativas del grupo control respecto al grupo de conejos obstruidos durante 15 y 90 días.

La variación del nivel de actina se muestra en la Figura 3. El análisis de la varianza demostró una variación significativa del peso vesical con el tiempo $(\mathrm{p}=0,000)$. El test de contraste a posteriori de Scheffe demostró diferencias significativas del grupo control respecto al grupo de conejos obstruidos durante 30 días.

La variación del nivel de actina sarcomérica se muestra en la Figura 4. El análisis de la varianza demostró una variación significativa del peso vesical con el tiempo $(\mathrm{p}=0,000)$. El test de contraste a posteriori de Scheffe demostró diferencias significativas del grupo control respecto al grupo de conejos obstruidos durante 30 y 180 días.

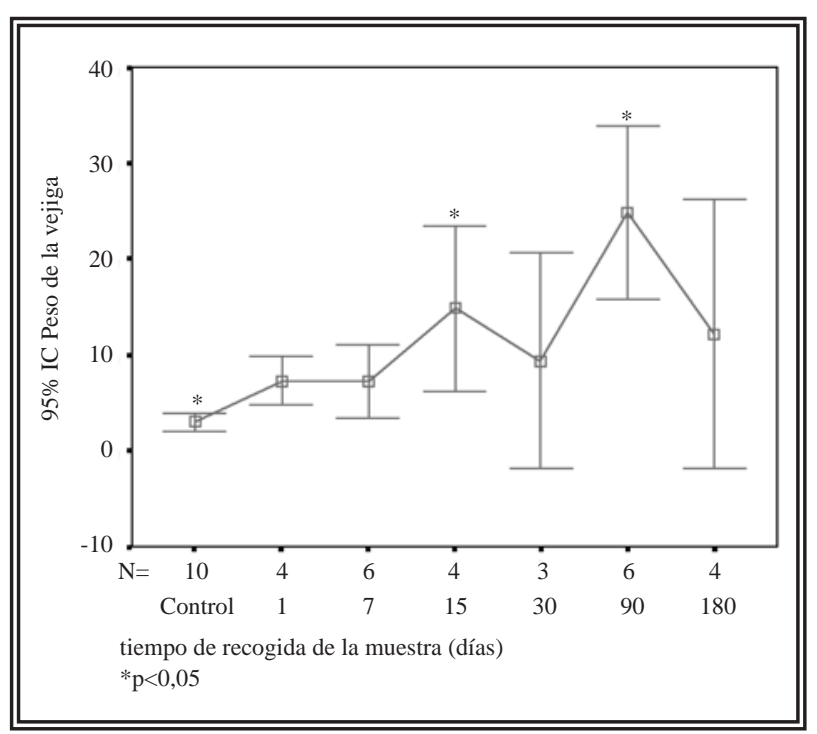

FIGURA 2. En ordenadas: peso de la vejiga en gramos. En abcisas se muestra el grupo control y los diferentes grupos experimentales, según el tiempo de duración de la obstrucción en dias. Cuadrado central: media, segmentos: $95 \%$ de confianza de la media (gráficos de error). Las diferencias entre los distintos valores del grupo obstruido fueron significativas a nivel global (ANOVA). El test de Sheffe demostró diferencias significativas del grupo control con los grupos de obstruidos durante 15 y 90 dias (asteriscos).

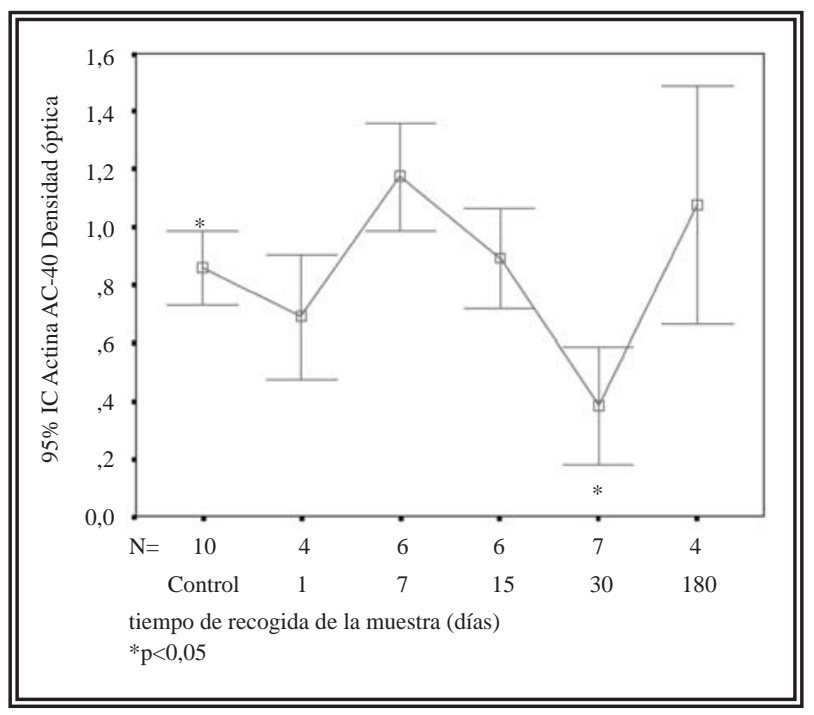

FIGURA 3. En ordenadas: densidad óptica (valor respecto a la vejiga control de 24 horas, que se toma como unidad). En abcisas se muestra el grupo control y los diferentes grupos experimentales, según el tiempo de duración de la obstrucción en días. Cuadrado central: media, segmentos: $95 \%$ de confianza de la media (gráficos de error). Las diferencias entre los distintos valores del grupo obstruido fueron significativas a nivel global (ANOVA). El test de Sheffe demostró diferencias significativas del grupo control con los grupos de obstruidos durante 30 y 180 dias (asteriscos).

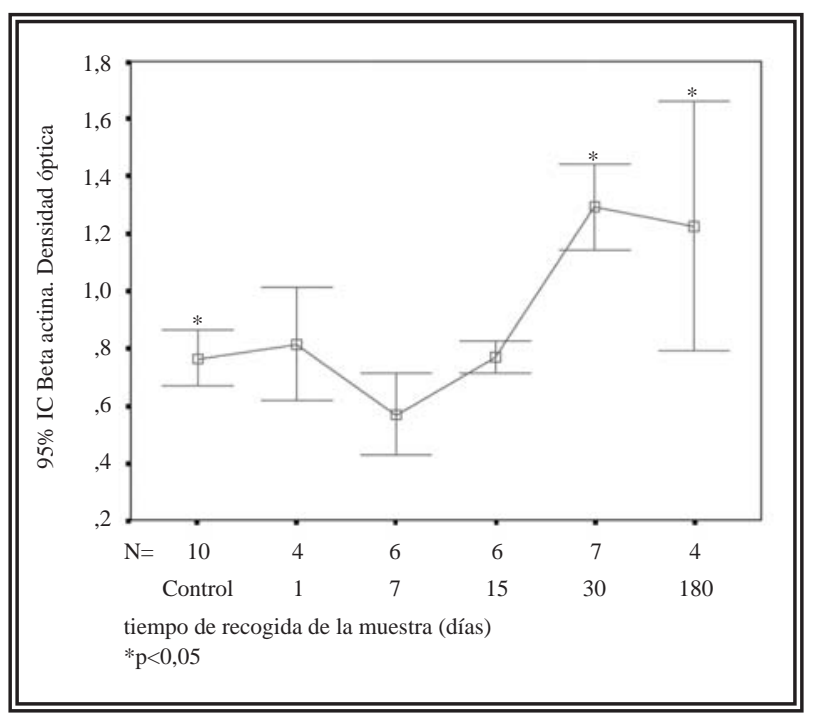

FIGURA 4. En ordenadas: densidad óptica (valor respecto a la vejiga control de 24 horas, que se toma como unidad). En abcisas se muestra el grupo control y los diferentes grupos experimentales, según el tiempo de duración de la obstrucción en días. Cuadrado central: media, segmentos: $95 \%$ de confianza de la media (gráficos de error). Las diferencias entre los distintos valores del grupo obstruido fueron significativas a nivel global (ANOVA). El test de Sheffe demostró diferencias significativas del grupo control con los grupos de obstruidos durante 30 dias (asteriscos). 
La variación del nivel de beta-actina se muestra en la Figura 5. El análisis de la varianza demostró una variación significativa del peso vesical con el tiempo $(\mathrm{p}=0,000)$. El test de contraste a posteriori de Scheffe demostró diferencias significativas del grupo control respecto al grupo de conejos obstruidos durante 7 días.

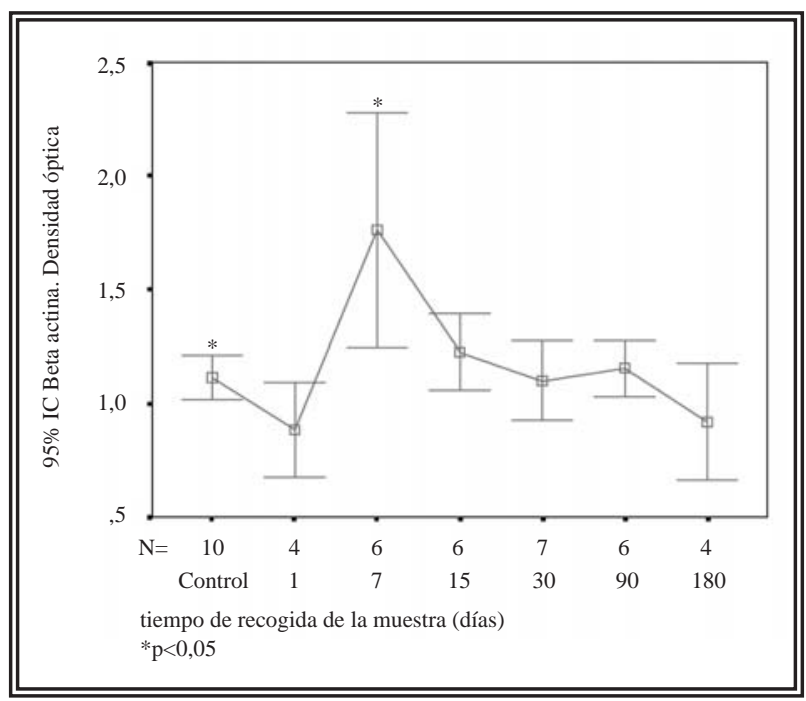

FIGURA 5. En ordenadas: densidad óptica (valor respecto a la vejiga control de 24 horas, que se toma como unidad). En abcisas se muestra el grupo control y los diferentes grupos experimentales, según el tiempo de duración de la obstrucción en dias. Cuadrado central: media, segmentos: $95 \%$ de confianza de la media (gráficos de error). Las diferencias entre los distintos valores del grupo obstruido fueron significativas a nivel global (ANOVA). El test de Sheffe demostró diferencias significativas del grupo control con los grupos de obstruidos durante 7 dias (asteriscos).

La variación del nivel de caldesmón se muestra en la Figura 6. El análisis de la varianza demostró una variación significativa del peso vesical con el tiempo $(\mathrm{p}=0,000)$. El test de contraste a posteriori de Scheffe demostró diferencias significativas del grupo control respecto al grupo de conejos obstruidos durante 15 días.

La variación del nivel de la proteína HSP-70 se muestra en la Figura 7. El análisis de la varianza demostró una variación significativa del peso vesical con el tiempo $(\mathrm{p}=0,000)$. El test de contraste a posteriori de Scheffe demostró diferencias significativas del grupo control respecto al grupo de conejos obstruidos durante 15 días.

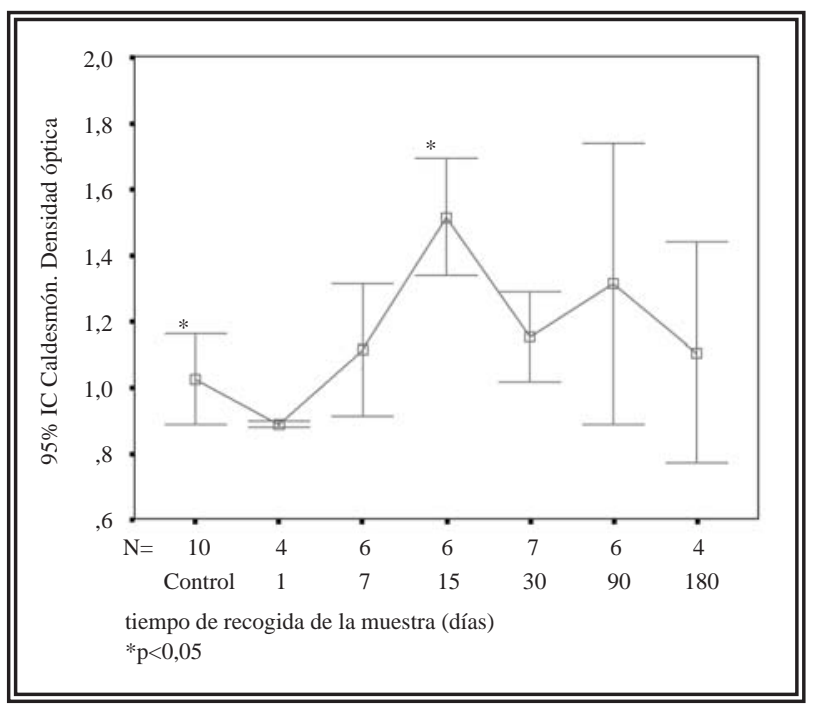

FIGURA 6. En ordenadas: densidad óptica (valor respecto a la vejiga control de 24 horas, que se toma como unidad). En abcisas se muestra el grupo control y los diferentes grupos experimentales, según el tiempo de duración de la obstrucción en días. Cuadrado central: media, segmentos: $95 \%$ de confianza de la media (gráficos de error). Las diferencias entre los distintos valores del grupo obstruido fueron significativas a nivel global (ANOVA). El test de Sheffe demostró diferencias significativas del grupo control con los grupos de obstruidos durante 7 dias (asteriscos).

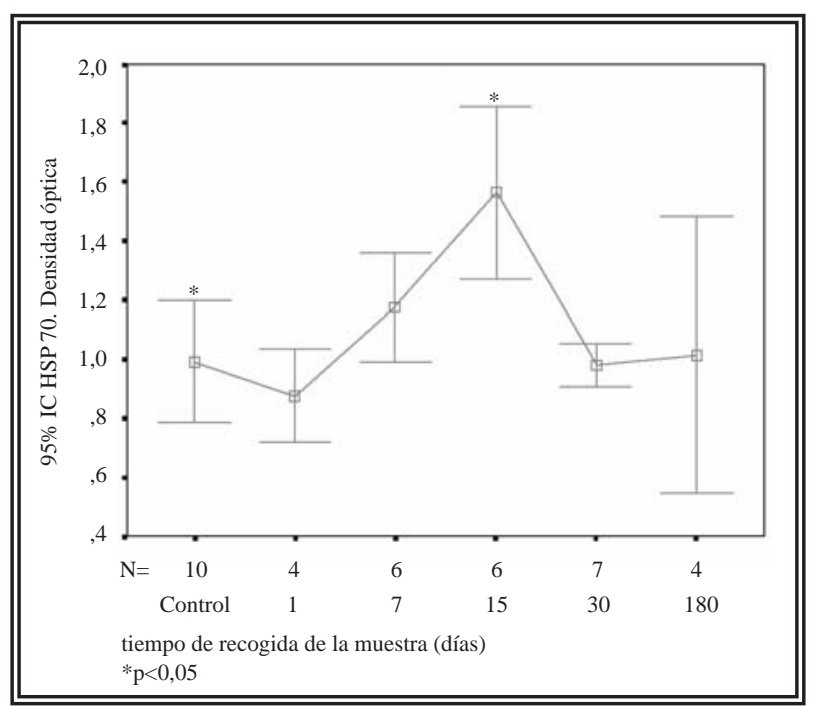

FIGURA 7. En ordenadas: densidad óptica (valor respecto a la vejiga control de 24 horas, que se toma como unidad). En abcisas se muestra el grupo control y los diferentes grupos experimentales según el tiempo de duración de la obstrucción en días. Cuadrado central: media, segmentos: $95 \%$ de confianza de la media (gráficos de error). Las diferencias entre los distintos valores del grupo obstruido fueron significativas a nivel global (ANOVA). El test de Sheffe demostró diferencias significativas del grupo control con los grupos de obstruidos durante 15 dias (asteriscos). 


\section{DISCUSIÓN}

De forma análoga a los cambios producidos por la hiperplasia benigna prostática en varones, la obstrucción urinaria experimental produce un aumento de la masa vesical. Este aumento no es uniforme, sino que transcurre en tres etapas características $^{7,13}$ :

- Una etapa inicial (entre 1 y 2 semanas), caracterizada por un aumento rápido de la masa muscular (hasta 10 veces). Histológicamente se caracteriza por hipertrofia de las células musculares e hiperplasia de la muscularis mucosae suburotelial.

- Una etapa de compensación, de duración variable, caracterizada por un aumento más lento de la masa vesical. En esta etapa prosigue la hipertrofia muscular y aparece tejido conectivo en la capa subserosa.

- Por último, una etapa de descompensación, caracterizada por un nuevo aumento brusco de la masa vesical (hasta 20 veces). En esta etapa el tejido muscular es reemplazado por tejido conectivo.

En nuestro estudio, el test de Scheffe, que compara el incremento del peso vesical en el grupo de conejos obstruidos, demostró diferencias significativas respecto al peso de la vejiga control a los 15 días y a los 90 días. El peso de las vejigas del grupo obstruido durante 15 días aumentó aproximadamente 5 veces comparado con el grupo control, mientras que el aumento del peso entre los 15 y 90 días fue aproximadamente sólo del doble.

Con estos datos podemos deducir que durante el tiempo que duró el estudio se cubrieron la etapa de aumento inicial del peso vesical (hasta la segunda semana) y de compensación (desde la segunda semana hasta su final, a los 3 meses).

No ha sido objetivo de nuestro estudio la miosina, dada la multiplicidad de trabajos sobre la misma en la obstrucción del tracto urinario inferior $^{9}$. Por el contrario, los trabajos sobre la actina son más escasos y contradictorios.

La actina constituye la mayor parte de las fibras lisas del tejido muscular. También forma parte del citoesqueleto (microtúbulos) de las células musculares y no musculares. Se conocen seis isoformas de actina, que se diferencian en la secuencia aminoácida del terminal amino (NH2), coincidiendo todas ellas en el terminar carboxilo $(\mathrm{COOH})$. Esas seis isoformas son: la alfa-actina sarcomérica del músculo esquelético, la alfa-actina del músculo cardiaco, la alfa-actina del músculo liso, la gamma-actina del músculo liso visceral y la gamma y beta-actina citoplasmáticas (no musculares) ${ }^{14}$. En nuestro estudio utilizamos tres tipos diferentes de anticuerpos para detectar tres tipos de actinas: un anticuerpo monoclonal que reacciona frente a todos los isotipos de actina, otro que reacciona específicamente frente a la beta-actina citoplasmática y un tercero que únicamente reacciona frente a la alfa-actina sarcomérica.

Según nuestros resultados los niveles de actina se mantienen estables durante todo el tiempo de estudio, excepto el día 30 que experimentan un descenso significativo. La beta-actina citoplasmática experimenta un incremento significativo el día 7, para luego volver a sus niveles basales. Por último, la alfa-actina sarcomérica experimenta un aumento de sus niveles a partir del día 30 que se mantienen hasta el final del estudio.

Otros autores, han encontrado que el nivel de actina se mantiene estable en las vejigas obstruidas, a diferencia del nivel de miosina que disminuye ${ }^{13,15}$. Sin embargo, se produciría una variación respecto al tipo de isoformas de actina, aumentando la concentración de gamma-acti$\mathrm{na}^{13,15-17}$ y disminuyendo la de beta-actina ${ }^{16,17}$, mientras que la alfa-actina permanecería invariable para algunos autores ${ }^{16,17}$, o disminuiría para otros $^{13,15}$. Durante la fase de hipertrofia muscular aumenta el nivel de gamma-actina, disminuyendo el nivel de la beta-actina ${ }^{16}$.

Sin embargo, durante la obstrucción vesical coexisten dos procesos: el de proliferación celular y el de diferenciación. Ambos procesos están inversamente relacionados. Debido a que las células maduras no conservan la capacidad para proliferar, es preciso su regresión a un fenotipo fetal, para volver a adquirir su potencial proliferativo, y posteriormente madurar y diferenciarse a células especializadas.

$\mathrm{El}$ aumento de los niveles de beta-actina al final de la primera semana, observado en nuestro estudio, podría explicarse por la diferenciación celular. Este incremento se asociaría a una disminución del nivel de actina a nivel del músculo 
liso ${ }^{18}$, responsable del descenso significativo del nivel total de actina, observado el día 30 de la obstrucción. La alfa-actina sarcomérica es específica del músculo esquelético, por lo que su aparición a partir del día 30 y su mantenimiento hasta el final del estudio podría explicarse por un nuevo proceso de desdiferenciación celular que podría evolucionar, esta vez, hacia la formación de tejido conectivo. Diversos estudios han demostrado que las células tumorales desdiferenciadas de origen estromal expresan alfa-actina sarcomérica $^{19,20}$. En cualquier caso, debido a la falta de especificidad de la actina, lo que hace que incluso la gamma-actina se presente en dos isoformas, una sola de las cuales es de origen muscular ${ }^{8}$, implica que esta proteína no es un buen marcador de las alteraciones musculares lisas durante la obstrucción del tracto urinario inferior.

El caldesmón forma parte, junto con la actina, de las fibras finas musculares. En nuestro estudio se comprobó un aumento de esta proteína que alcanzó un máximo el día 15 de la obstrucción, para descender posteriormente a sus valores basales. Esta elevación coincidiría con la primera etapa de la obstrucción caracterizada por la hipertrofia muscular.

Otros autores, también comprueban la elevación de los niveles de caldesmón a las dos semanas de la obstrucción ${ }^{21}$. Este incremento se produciría principalmente a expensas de la forma fetal del caldesmón o 1-caldesmón, mientras que la forma madura o h-caldesmón disminuiría ${ }^{22} \mathrm{o}$ permanecería igual $^{21}$. Este proceso sería una faceta más del fenómeno de desdiferenciación celular ${ }^{9}$. Los niveles de caldesmón fetal (1-caldesmón), se normalizan rápidamente tras la liberación de la obstrucción, por lo que es posible que esta proteína sea un buen marcador de reversibilidad de la función muscular después de la desobstrucción ${ }^{23}$.

Por último, la expresión de la proteína de stress celular HSP-70, mostró una elevación a partir de la primera semana de obstrucción, aunque sus niveles no fueron significativos hasta los 15 días de la obstrucción. Otros autores también comprueban una elevación de esta proteína de stress después de una obstrucción parcial. Sin embargo, los valores máximos se alcanzan mucho antes (a las 24 horas), para normalizarse a la semana ${ }^{24}$. La principal desventaja de esta proteína como marcador de obstrucción es que su expresión es inespecífica del proceso causal. Aparece ante cualquier agresión tisular como el calor $^{25}$ o la simple distensión vesical ${ }^{26}$.

En conclusión, de todas las proteínas analizadas, únicamente el caldesmón presentó unos datos consistentes tanto con la evolución de la obstrucción, como con los presentados por otros autores, por lo que podría ser un marcador útil de obstrucción del tracto urinario inferior. Sin embargo, son precisos nuevos estudios para comprobar su utilidad clínica.

Agradecimientos: Queremos expresar nuestro reconocimiento por su valiosa ayuda a la Doctora Sánchez de la Muela, Profesora titular de Cirugía de la Facultad de Veterinaria de la Universidad Complutense de Madrid.

\section{REFERENCIAS}

1. LEE A, RUSSELL EB, GARRAWAY WM, PRESCOTT RJ.: Three year follow-up of a comunity-based cohort of men with untreated benign prostatic hyperplasia. Eur Urol 1996; 30: 11-17.

2. SHERIF M, BLAIVAS J.: Functional types of prostatic obstruction. Neurourol Urodyn 1988; 7: 221-222.

3. ROSIER P, DE WILDT MJAM, DE LA ROSETTE JJ, DEBRUYNE FM, WIJKSTRA H.: Analysis of maximum detrusor contraction power in relation to bladder emptying in patients with lower urinary tract symptoms and benign prostatic enlargement. $J$ Urol 1995; 154 (6): 2.137-2.142.

4. BOSCH JLH, KRANSE R, VAN MASTRIGT R, SCHROEDER F.: Dependence of male efficiency on age, bladder contractility and urethral resistance: development of a voiding efficiency nomogram. $J$ Urol 1995; 154 (1): 190194.

5. SULLIVAN M, YALLA S.: Detrusor contractility and compliance characteristics in adult male patients with obstructive and nonobstructive voiding dysfunction. J Urol 1996; 155: 1.995-2.000.

6. GUAN, KIRULUTA G, COOLSAET B, ELHILAHI M.: A minipig model for urinary evaluation of intravesical obstruction and its possible reversibility. J Urol 1995; 154: 580-585.

7. LEVIN R, LONGHURST P, MONSON F, KATO K, WEIN A.: Effect of bladder outlet obstruction on the morphology, physiology ans pharmacology of the bladder. Prostate 1990; 3 supp: 926.

8. MALMQVIST U, ARNER A, UVELIUS B.: Cytoskeletal and contractile proteins in detrusor smooth muscle from bladders with outlet obstruction. A comparative study in rat and man. Scandinavian Journal of Urology and Nephrology 1991; 25: 261267.

9. ZIMMERN P, LIN V, MCCONNELL JD.: Smooth-muscle physiology. Urol Clin North Am 1996; 23: 211 219 . 
10. ZHAO Y, LEVIN RM, MONSON FC, CHACKO S.: Expression of constitutive heat shock protein-70 in normal (non-stressed) rabbit urinary bladder tissue. Mol Cell Biochem 1993 apr 7; 121 (1): 1319.

11. BRADFORD M.: A rapid and sensitive method for the quantitation of microgram quantities of protein utilizing the principle of protein-dye binding. Anal Biochem 1976; 72: 248-254.

12. BURNETTE WN.: "Western Blotting": electrophoretic transfer of proteins from sodium dodecyl sulfatepolyacrylamide gels to unmodified nitrocellulose and radiographic detection with antibody and radioiodinated protein. Anal Biochem 1981; 112 : 195-203.

13. LEVIN RM, MONSON FC, HAUGAARD N, BUTTYAN R, HUDSON A, ROELOFS M., SARTORE S, WEIN AJ.: Genetic and cellular characteristics of bladder outlet obstruction. Urol Clin North Am 1995; 22: 263-283.

14. CHACKO S, DISANTO M, WANG Z, ZDERIC S, WEIN AJ.: Contractile protein changes in urinary bladder smooth muscle during obstructioninduced hypertrophy. Scan J Urol Nephrol Supp 1997; 184: 67-76.

15. MALMQVIST U, ARNER A, UVELIUS B.: Contractile and cytoskeletal proteins in smooth muscle during hypertrophy and its reversal. Am J Physiol 1991 may; 260 (5 Pt 1): C1085-93.

16. KIM Y, WANG Z, LEVIN R, CHACKO S.: Alterations in the expression of the beta-cytoplasmatic and gamma-smooth muscle actins in hypertrophied urinary bladder smooth muscle. Mol Cell Biochem 1994; 131: 115-124.

17. CHACKO S, LONGHURST PA.: Regulation of actomyosin and contraction in smooth muscle. World $J$ Urol 1994; 12 (5): 292-297.

18. OWENS GK, LOEB A, GORDON D, THOMPSON MM.:Expression of smooth muscle-specific alphaisoactin in cultured vascular smooth muscle cells: relationship between growth and cytodiferentiation. J Cell Biol 1986; 102: 303-306.
19. SAPPINO AP, SKALLI O, JACKSON B, SCHURCH W, GABBIANI G.: Smooth-muscle differentiation in stromal cells of malignant and non-malignant breast tissues. Int $J$ Cancer 1988 may 15; 41 (5): 707-712.

20. SCHURCH W, SKALLI O, SEEMAYER TA, GABBIANI G.: Intermediate filament proteins and actin isoforms as markers for soft tissue tumour differentiation and origin. I. Smooth muscle tumours. Am J Pathol 1987 jul; 128 (1): 91-103.

21. ZHANG E, STEIN R, ZDERIC S, WEIN AJ, CHACKO S.: Overexpression of non-muscle isoform caldesmon. A protein associated with stress fibers, in detrusor remodeling following outlet obstruction. $J$ Urol 2001; 165 (5 supp): 248.

22. DISANTO M, MENON C, WEIN AJ, CHACKO S.: Changes in the composition of myosin isoforms and caldesmon in the detrusor muscle following partial outlet obstruction. Neurourol Urodyn 1998; 17: 444-445.

23. BURKHARD FC, LEMACK GE, ZIMMERN PE, LIN VK, MCDONNELL JD.: Contractile protein expression in bladder smooth muscle is a marker of phenotypic modulation after outlet obstruction in the rabbit model. J Urol 2001; 165: 963-967.

24. ZHAO Y, CHACKO S, LEVIN R.: Expression of stress proteins (HSP-70 and HSP-90) in the rabbit urinary bladder subjected to partial outlet obstruction. Mol Cell Biochemistry 1994; 130: 49-55.

25. CHEN MW, KRASNAPOLSKY L, LEVIN RM, BUTTYAN R.: An early molecular response induced by acute overdistension of the rabbit urinary bladder. Mol Cell Biochem 1994 mar 16; 132 (1): 39-44.

26. CURRIE RW, WHITE FP.: Characterization of the synthesis and accumulation of a 71-kilodalton protein induced in rat tissues after hyperthermia. Can J Biochem Cell Biol 1983 jun; 61 (6): 438-446.

Dr. J. Salinas Casado

C/ Solano, 6 - 4ํD

28223 Prado de Somosaguas (Madrid)

(Trabajo recibido el 10 abril de 2003) 